\title{
EDIFİCATE
}

I Congreso de Escuelas de Edificación y Arquitectura Técnica de España

València, 4 y 5 de noviembre de 2021

Escuela Técnica Superior de Ingeniería de Edificación

Universitat Politècnica de València

Doi: https://doi.org/10.4995/EDIFICATE2021.2021.13585

\section{Introducción de nuevas estrategias para el aprendizaje autónomo en alumnos de primer curso}

\section{Implementating new strategies for autonomous learning in first-year students}

\author{
María-Isabel Giner-Garcíaa , Ángeles Rodrigo-Molina ${ }^{b}$ \\ ${ }^{a}$ Escuela Técnica Superior de Ingeniería de Edificación, Universitat Politècnica de València, Camino de \\ Vera s/n, 46022, Valencia (España). magigar@csa.upv.es. \\ ${ }^{\mathrm{b}}$ Escuela Técnica Superior de Ingeniería del Diseño, Universitat Politècnica de València, Camino de \\ Vera s/n, 46022, Valencia (España). arodrigo@ega.upv.es
}

\begin{abstract}
University training is more than the academic development of the student. The acquisition of certain competences, the know-how in specific situations applying knowledge, as well as skills and attitudes are also essential in the learning process. The paper shows the educational strategy implemented involving first-year students. The goal is that the students learn skills and resources destined to organise and to plan themselves facing the autonomous work. In addition, they improve their academic performance. Due mainly to the inexperience of these students, their difficulties for a project where they must work in an autonomous way and that it requires a planning in time have been evident. The proposed strategies set out a certain number of support guidelines for autonomous learning, which allow to improve the results. To this end, a work planning is carried out, and assessed tutorials are scheduled, where in each one of them the items marked in the calendar must be presented. Tools are used to analyse both the functioning and usefulness of the procedure, and the improvement in learning results.
\end{abstract}

Keywords: autonomous learning, educational strategies, planning, tutorial, architectural graphic expression, architectural drawing. 


\begin{abstract}
Resumen
La formación universitaria es algo más que el desarrollo académico del alumno. La adquisición de ciertas competencias, el saber hacer ante situaciones concretas aplicando conocimientos, así como las habilidades $y$ actitudes, son también esenciales en el proceso de aprendizaje. El trabajo que se expone muestra la estrategia educativa puesta en marcha con alumnos de primer curso. El objetivo es que aprendan habilidades y recursos destinados a organizarse y planificarse ante el trabajo autónomo, mejorando así su rendimiento académico. Debido principalmente a la inexperiencia de estos alumnos, se han evidenciado sus dificultades ante un proyecto donde deban trabajar de forma autónoma y que requiera una planificación en el tiempo. Las estrategias planteadas marcan una serie de pautas de apoyo para el aprendizaje autónomo, y permiten mejorar los resultados. Para ello se realiza una planificación del trabajo y se programan unas tutorías evaluables, donde en cada una de ellas se deben presentar los items marcados en calendario. Se utilizan herramientas de análisis tanto del funcionamiento $y$ utilidad del procedimiento, como de la mejora en los resultados aprendizaje.
\end{abstract}

Palabras clave: aprendizaje autónomo, estrategias educativas, planificación tutorias, expresión gráfica arquitectónica, dibujo arquitectónico. 


\section{Introducción}

Expresión Gráfica es una asignatura troncal, que se imparte en el primer curso de Grado de Arquitectura Técnica. En ella se introduce al alumno en el lenguaje gráfico y en el levantamiento arquitectónico. Es una asignatura anual, con tres horas de clase a la semana, en su mayoría prácticas que se realizan fuera del aula. Durante las nueve últimas semanas del curso se desarrolla un proyecto: levantamiento gráfico de un edificio o un conjunto arquitectónico de pequeñas dimensiones. Este proyecto se realiza fuera del horario lectivo, aunque se establece alguna sesión de trabajo en clase, y una serie de tutorías. El objetivo de la realización de este proyecto es fomentar el aprendizaje autónomo del alumno, trabajando en un caso real donde se deben aplicar los conocimientos adquiridos en el aula. El proyecto puede realizarse de forma individual, o por equipos (a elección de los propios alumnos).

El motivo de implantar una nueva estrategia de aplicación para el desarrollo del proyecto, fue el análisis de los resultados académicos de los últimos años. Se detectó una disminución de la calidad de los trabajos, así como un aumento en el número de trabajos no presentados. El porcentaje de trabajos no finalizados aumentó del $5 \%$ el primer año, al 6 ' $25 \%$ el segundo, y al 8 ' $10 \%$ en el último.

Igualmente se analizaron las posibles causas, y se evaluaron una serie de hipótesis:

La primera causa a considerar fue la dimensión del proyecto, probablemente demasiado extenso en cuanto al contenido en relación con el tiempo dado para su ejecución. No obstante, también existe un elevado porcentaje de alumnos que terminan puntualmente el trabajo con unos buenos e incluso excelentes resultados, por lo que se consideró que éste no era el factor principal de aumento de los malos resultados.

La segunda causa analizada fue el aumento del número de trabajos a realizar por el alumno en las diferentes asignaturas del curso académico. Esta cuestión requiere un análisis más profundo en cuanto a la transversalidad horizontal entre asignaturas de un mismo curso (Montes, 2011). Aún así, fue tomada en consideración, y se modificaron las fechas, tanto de inicio como de entrega final del proyecto, a fin de evitar la superposición excesiva de entregas.

Otro motivo que influiría en los malos resultados es la no utilización por parte del alumno de las tutorías. Todo trabajo autónomo requiere ser tutorizado, y aunque, en este caso, se establecen tres sesiones de clase para el seguimiento del mismo, es totalmente imprescindible el uso de las tutorías. La orientación del profesor a corto plazo ayuda al alumno a avanzar con mayor claridad y seguridad, y permite el desarrollo integral del alumno (Bisquerra, 2002). Por tanto, se consideran las tutorías uno de los puntos importantes a reflexionar.

Por último, otra problemática, que se viene observando durante los últimos años, es la carencia de destreza y las dificultades en las que se encuentran, sobretodo (los alumnos de primer curso) para organizarse y planificarse a medio y largo plazo. 
De todo lo anterior se obtiene como resultado que las dificultades más destacables surgen de la inexperiencia del alumnado a trabajar de forma autónoma, así como de la carencia de recursos para la planificación y organización frente a un trabajo de varios meses, agravado por la falta de uso de la tutoría para decibir el feedback del profesor. Por lo que, se decide profundizar en estos aspectos.

\section{Objetivos y resultados de aprendizaje}

Los objetivos de aprendizaje que se marcaron fueron: por un lado, impulsar estrategias y procedimientos que fomenten la motivación, la autonomía en el aprendizaje, y la participación del alumnado; por otro, asegurar la orientación personal que responda a las necesidades de los alumnos; y por último, promover estrategias que guíen al alumno para planificar sus trabajos.

En cuanto a los resultados de aprendizaje que se pretendían alcanzar utilizando estas estrategias fueron: ser capaz de planificar a medio-largo plazo un proyecto de relativa complejidad; identificar y describir los diferentes problemas que pueden surgir a la hora de realizar un levantamiento gráfico; y resolver, mediante los recursos adquiridos, cuestiones de la forma más conveniente.

Por ello, dichas estrategias se centraron en la incorporación de una planificación, corealizada alumno/profesor que permitiese alcanzar los ítems marcados en el trabajo, así como una serie de tutorías, obligatorias y evaluables, introducidas dentro de dicha planificación.

Con la incorporación de estas tutorías, se pretende incrementar y fortalecer las habilidades para consultar, analizar y seleccionar información aplicable a la enseñanza y aprendizaje del dibujo arquitectónico en contextos específicos. De este modo, se fortalece el desarrollo de hábitos de trabajo que favorezcan el crecimiento personal y el descubrimiento de nuevas formas de adquisición del conocimiento (Bisquerra, 2012). Se pretende trabajar tanto las estrategias cognitivas como las metacognitivas. Para las estrategias cognitivas, es importante tener claro el concepto de cognición, entendido como la acción de conocer, es decir recoger, organizar y utilizar el conocimiento (Gellalty, 1997). Las estrategias metacognitivas implican planificar qué se va aprender, y evaluar logros que les permitan concretar aprendizajes.

\section{Desarrollo de la innovación y metodología}

En consecuencia, se incorporó al proyecto una serie de recursos encaminados a conseguir los objetivos y resultados de aprendizaje. Una vez conocido el proyecto a desarrollar y el tiempo que se disponía para su ejecución, se procedió a la realización de un organigrama de trabajo (Tabla 1), incluyendo en él las tutorías evaluables. Dicha planificación, como se comentaba anteriormente, se trabajó conjuntamente con el alumnado. 
Tabla 1. Resumen del organigrama de trabajo

\begin{tabular}{|c|c|c|c|c|}
\hline Tareas & Descripción & Lugar & Duración & Responsable \\
\hline 1 & $\begin{array}{l}\text { Presentación proyecto, } \\
\text { metodologia de trabajo }\end{array}$ & Aula & $45 \mathrm{~min}$ & profesora \\
\hline 2 & $\begin{array}{l}\text { Visita edificio / trabajo de } \\
\text { campo }\end{array}$ & Edificio & $1 \mathrm{~h} 15 \mathrm{~min}$ & alumno/profesor \\
\hline 3 & Planificación/organigrama & Aula & $1 \mathrm{~h}$ & alumno/profesor \\
\hline 4 & Formacion de grupos & casa & $-\cdots$ & alumno \\
\hline 5 & Desarrollo ítem 1 & Edificio/casa & 3 semanas & alumno \\
\hline 6 & $\begin{array}{l}\text { 1a Sesión tutorías. Entrega } \\
\text { ficha seguimiento }\end{array}$ & Aula & $30 \mathrm{~min} /$ equipo & alumnos/profesor \\
\hline 7 & Desarrollo ítem 2 & Edificio/casa & 2 semanas & alumno \\
\hline 8 & Sesión aula & Aula & 3 horas & alumno/ tutor \\
\hline 9 & $\begin{array}{l}\text { 2a Sesión tutorías. Entrega } \\
\text { ficha seguimiento }\end{array}$ & Aula & $30 \mathrm{~min} /$ equipo & alumnos/profesor \\
\hline 10 & Desarrollo ítem 3 & Edificio/casa & 2,5 semanas & alumno \\
\hline 11 & Sesión aula & Aula & 3 horas & alumno/ tutor \\
\hline 12 & $\begin{array}{l}\text { 3a Sesión tutorías. Entrega } \\
\text { ficha seguimiento }\end{array}$ & Aula & $30 \mathrm{~min} /$ equipo & alumnos/profesor \\
\hline 13 & Entrega final & Aula & 2,5 semanas & alumnos/profesor \\
\hline 14 & Calificaciones & Web & 1 semana & profesor \\
\hline
\end{tabular}

Por otro lado, se integraron diversas herramientas de análisis de las tutorías (Badillo, 2007), con el fin de recoger evidencias que permitiesen detectar defectos de organización. Se plantearon herramientas como: el "registro de asistencia"; "informe tutor" -evalúa logro de ítem-; y "sondeo/encuesta", a completar por el alumno, donde se valoran aspectos positivos y negativos de factores como la organización y la eficacia de las tutorías, así como de la ayuda para su planificación. También se introdujeron herramientas de organización -"fichas de seguimiento" (Fig. 1)- a cumplimentar por el alumnado, y que permiten comprobar el cumplimiento de la planificación.

El proyecto consistió en el levantamiento gráfico de un edificio, donde se muestraban todas las formas reales y dimensiones, aplicando los criterios gráficos desarrollados en la asignatura y normativa vigente. Duración 9 semanas. La Metodología seguida para el desarrollo fue la siguiente: 
1.- Los alumnos optarán por trabajar de forma individual o en equipo ( $2 / 3$ alumnos por equipo). Si se decide trabajar en equipo (el trabajo es colaborativo), deberá haber una coordinación entre ellos para realizar la diferente planimetría.

2.- Se realizarán tres tutorías grupales evaluables, una al inicio, otro a mitad, y una última al final del trabajo. Si se trabaja en equipo deberán acudir conjuntamente todos los miembros.

3.- La planificación del proyecto, tiempos de trabajo, ítems a alcanzar...., se realizará de forma conjunta entre los alumnos y el profesor.

3.- En cada tutoría los alumnos deberán aportar, además de los planos a analizar y revisar, una ficha de seguimiento (Fig. 1) del trabajo acometido hasta el momento (ítems alcanzados, problemas surgidos y dudas).

\begin{tabular}{|l|l|}
\hline \multirow{2}{*}{ Nombre alumnos: } & Alumno 1: \\
\cline { 2 - 2 } & Alumno 2: \\
\hline
\end{tabular}

Rellena con una cruz los items alcanzados

\begin{tabular}{|r|c|l|l|}
\hline \multicolumn{1}{|c|}{ Ítems } & Distribución trabajo & Ítem resuelto en fecha & Observaciones/ dificultades encontradas \\
\hline Croquis entorno acotado la revisión: Jueves 25 de abril & $\begin{array}{c}\text { Alumno 1 } \\
\text { Alumno 2 }\end{array}$ & & \\
\hline Croquis planta baja & Alumno 1 & & \\
\hline Croquis planta primera & Alumno 2 & & \\
\hline Sección Vertical 1 & Alumno 1 & & \\
\hline Sección Vertical 2 & Alumno 2 & & \\
\hline Alzado principal & Alumno 1 & & \\
\hline Alzado Posterior & Alumno 2 & & \\
\hline Perfil izquierdo & Alumno 1 & & \\
\hline Perfil derecho & Alumno 2 & & \\
\hline Cuestiones a preguntar en la tutoria 1: & &
\end{tabular}

Fig. 1. Modelo de ficha de seguimiento.

4.- Se facilita a los alumnos la "ficha de autoevaluación" (Fig. 2) que permite la evaluación de cada uno de los ítems, y que será la misma que utilizará la profesora.

5.- Evaluación: - El trabajo supone un $20 \%$ de la nota final de curso. - Las tutorías, la asistencia, logro de ítems en fecha, cumplimentación de fichas de seguimiento, y modificación en plano de las correcciones hechas por la profesora, puntuarán $10 \%$ sobre la nota final.

En la primera sesión, una vez analizado el enunciado del proyecto, y conocido el edificio objeto de estudio, se realiza el organigrama de trabajo. Éste se realiza teniendo en cuenta el volumen de trabajo, tiempo para el desarrollo de cada de las partes del proyecto, y posibles contratiempos que los alumnos pudiesen tener. En él, además, se indica el calendario de las tutorías, y los ítems a alcanzar para cada una de ellas. Estas tutorías permiten marcar un ritmo de trabajo para lograr objetivos concretos, en un periodo de tiempo adecuado. Permiten observar el proyecto en actividades concretas alcanzables. 
Analiza cada una de las partes del ejercicio plantado atendiendo a los siguientes criterios:

\begin{tabular}{|l|l|}
\hline A: Muy bien & D: Regular \\
\hline B: Bastante bien & E: Mal \\
\hline C: Bien & F: No entiendo lo que me preguntan \\
\hline
\end{tabular}

\begin{tabular}{|c|c|c|c|c|c|c|}
\hline SISTEMA DIÉDRICO & A & B & C & D & E & $\mathbf{F}$ \\
\hline \multicolumn{7}{|l|}{ № de vistas necesarias para la definición completa del elemento } \\
\hline \multicolumn{7}{|l|}{ Proyección correcta de vistas } \\
\hline \multicolumn{7}{|l|}{ Abatimiento correcto de vistas } \\
\hline \multicolumn{7}{|l|}{ Correspondencia entre todas las vistas } \\
\hline \multicolumn{7}{|l|}{ Distribución correcta de las vistas dentro del papel } \\
\hline \multicolumn{7}{|l|}{ Definición (análisis) correcto de las vistas } \\
\hline \multicolumn{7}{|l|}{ Correcta denominación de cada vista } \\
\hline \multicolumn{7}{|l|}{ Valora de forma general el sistema diédrico de tu croquis } \\
\hline GRAFISMO - VALORACIÓN DE LÍNEAS - ROTULACIÓN & A & B & C & D & E & $\mathbf{F}$ \\
\hline \multicolumn{7}{|l|}{ Correcto dibujado de los trazos (precisos y uniformes) } \\
\hline \multicolumn{7}{|l|}{ Valoración de líneas auxiliares } \\
\hline \multicolumn{7}{|l|}{ Valoración de aristas proyectadas } \\
\hline \multicolumn{7}{|l|}{ Valoración de aristas seccionadas } \\
\hline \multicolumn{7}{|l|}{$\begin{array}{l}\text { Correcta ubicación y grafiado de los planos de corte/planos de } \\
\text { sección }\end{array}$} \\
\hline \multicolumn{7}{|l|}{ Correcta ubicación y grafiado de los ejes de simetria } \\
\hline \multicolumn{7}{|l|}{ Situación de la línea de tierra } \\
\hline \multicolumn{7}{|l|}{ Correcto grafismo de los límites del dibujo } \\
\hline \multicolumn{7}{|l|}{$\begin{array}{l}\text { Rotulación correcta del nombre de cada vista, homogénea y } \\
\text { proporcionada }\end{array}$} \\
\hline \multicolumn{7}{|l|}{$\begin{array}{l}\text { El cajetín está situado correctamente, y se han rellenado todos } \\
\text { sus apartados }\end{array}$} \\
\hline \multicolumn{7}{|l|}{ Correcta rotulación del cajetín } \\
\hline $\begin{array}{l}\text { Valora de forma general el grafismo, la valoración de líneas y la } \\
\text { rotulación de tu croquis. }\end{array}$ & & & & & & \\
\hline
\end{tabular}

Fig. 2. Ficha autoevaluación.

Tras cada tutoría y como evidencia de su desarrollo, la profesora realiza un informe (Fig. 3) de cada uno de los equipos de trabajo. En él se analizarán los ítems alcanzados, la calidad del trabajo, las dudas y soluciones aportadas.

\begin{tabular}{|c|c|c|c|}
\hline items REALIZADOS & st & No & OBSERVACIONES \\
\hline \multicolumn{4}{|l|}{ Croquis entorno acotado } \\
\hline \multicolumn{4}{|l|}{ Croquis planta baja } \\
\hline \multicolumn{4}{|l|}{ Croquis planta primera } \\
\hline \multicolumn{4}{|l|}{ Sección Vertical 1} \\
\hline \multicolumn{4}{|l|}{ Sección Vertical 2} \\
\hline \multicolumn{4}{|l|}{ Alzado principal } \\
\hline \multicolumn{4}{|l|}{ Alzado Posterior } \\
\hline \multicolumn{4}{|l|}{ Perfil zquierdo } \\
\hline Perfil derecho & & & \\
\hline ade seguimiento & & & \\
\hline
\end{tabular}

\begin{tabular}{|l|l|}
\hline ANÁLISIS \\
\hline SISTEMA DIÉRRICO & \\
\hline $\begin{array}{l}\text { ANÁLSIS DE FORMAS/ } \\
\text { PROPORCION }\end{array}$ & \\
\hline VALORACIÓN DE LINEA & \\
\hline GRAFISMO & \\
\hline OTROS & \\
\hline
\end{tabular}

Fig. 3. Modelo para el informe del profesor donde se indican ítems alcanzados por cada equipo, análisis del trabajo realizado, funcionamiento y organización. 


\section{Resultados}

Tras la entrega, y antes de conocerse los resultados, se organizó una sesión a la cual asistieron la totalidad de los alumnos. En ella se plantea una encuesta con diferentes cuestiones a fin de conocer la opinión del alumnado (Fig. 4), ventajas e inconvenientes tanto de la planificación como de las tutorías llevadas a cabo.

En referencia a la planificación se valoró muy positivamente el poder alcanzar ítems parciales, por tanto metas a corto plazo asequibles en el tiempo. Esto les permitía trabajar de forma más sosegada, y aminorar la presión del proyecto íntegro. En su mayoría se ven capaces de enfrentarse a un trabajo de estas características, y planificar las tareas de forma adecuada en el tiempo. Por otro lado, si en un principio les pareció tedioso el desarrollo de la planificación, posteriormente la consideraron imprescindible para poder alcanzar los objetivos de un proyecto de estas características.

Por lo que respecta a las tutorías, se comentó que ayudaron a reflexionar acerca del progreso y desarrollo del proyecto. Les permitió mejorar y modificar errores importantes que mejoraran su calificación final. En cuanto a opiniones negativas, la obligatoriedad de las mismas por tener una fecha inamovible en el calendario.

De forma generalizada se continúa pensando que el proyecto es excesivamente extenso.

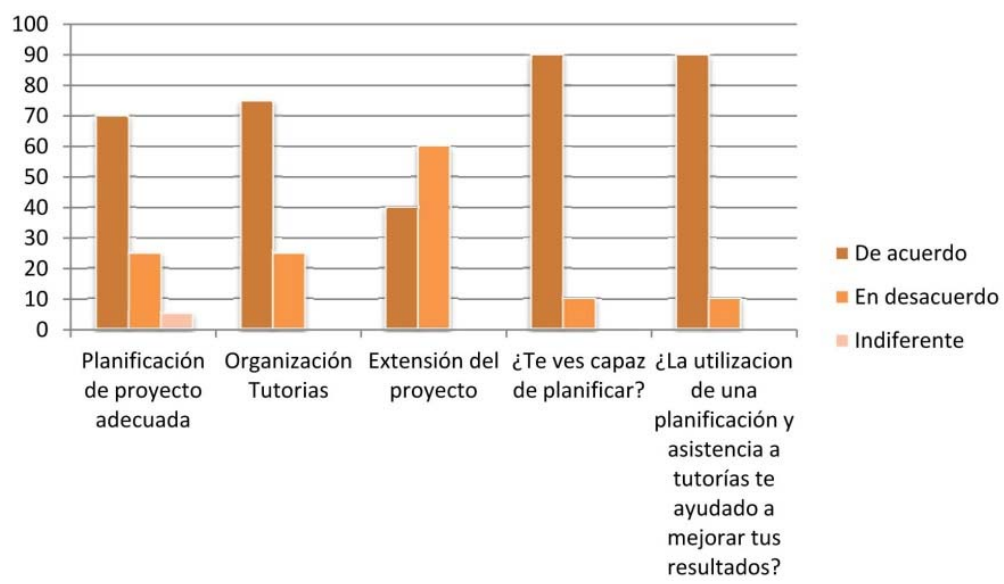

Fig. 4. Resultados de encuesta de opinión realizada a los alumnos.

Respecto a la colaboración y participación de los alumnos, y el trabajo en equipo. Informe del profesorado.

De los 15 alumnos, 7 decidieron trabajar de forma individual (1 abandonó la asignatura antes de empezar el trabajo), y los 8 restantes, decidieron trabajar en equipo de 2 (ninguno de 3 miembros, aconsejado por la profesora).

Los alumnos que trabajaron de forma individual en sus inicios se quejaron del volumen de trabajo, ya que era igual al realizado por los que trabajaban en equipo. Los alumnos que 
trabajaron en equipo, tuvieron problemas de coordinación, ya que en casi todas las tareas dependían de sus compañeros. De forma general, se considera muy satisfactoria la colaboración y participación entre los alumnos.

Respecto al funcionamiento de las tutorías. Informe del profesorado.

Problemas: Los días estaban establecidos en la planificación pero no hora exacta, ya que dependía del número de equipos que se formasen. Estas se difundieron a través del correo electrónico sin tener la confirmación de su lectura. Esto ocasionó que en la primera sesión algún grupo no se presentase, y se tuvo que fijar otra fecha. Evidentemente la solución pasa por asignar las horas en clase.

Por otro lado, el tiempo fijado para cada equipo de 30 minutos, en muchos casos se quedaba escaso. Muchos de los equipos demandaron más tutorías fuera de las establecidas como obligatorias.

Respecto a los resultados de aprendizaje. Informe del profesorado.

En las tutorías se pudo comprobar el avance de los alumnos a la hora de identificar problemas, analizarlos y solucionarlos de la mejor manera posible utilizando recursos gráficos adecuados. Por otro lado, durante el transcurso del proyecto algunos grupos tuvieron que modificar la planificación y adaptarla a sus necesidades. Si bien no tenemos posibilidad de comprobar de forma fehaciente si los alumnos son capaces de planificar otro proyecto de similares características, en esta asignatura, el hecho de saber acomodar, reorganizar y modificar la planificación a sus circunstancias es un indicador de su capacidad para realizarla.

Respeto a los resultados curriculares, el $100 \%$ de los alumnos aprobaron el trabajo en este grupo, y más de un $70 \%$ obtuvieron una nota superior a 7 .

\section{Conclusiones}

El problema principal surgido es la demanda del tiempo necesario para poder realizar correctamente todas las tutorías. Cierto es que, algunos equipos han necesitado más del doble del tiempo establecido. Esta problemática se podría solucionar de dos formas. Por un lado, simplemente centrando los temas a desarrollar durante la tutoría, y no desviándose de los mismos. Si surgieran dudas fuera de los ítems establecidos se podrían concertar tutorías particulares fuera de las establecidas en las tareas.

Por otro lado, otra solución sería aumentar el número de alumnos en cada tutoría. La experiencia ha demostrado que se podría hacer una revisión grupal con unos 6 alumnos, ya que los problemas que les surgen son comunes y muy repetitivos.

Por otro lado, además de resolver los problemas particulares de cada alumno, escuchar y solucionar las dudas y cuestiones que surgen a otros compañeros, también es muy ventajoso en su aprendizaje. Se podría incluso introducir la coevaluación (Lillo, 2013) dentro de las tutorías. 
De forma general, la experiencia ha sido muy positiva como muestra el $100 \%$ de aprobados. Esto demuestra que, aunque el aprendizaje autónomo en alumnos de primer curso es más complicado debido a su poca experiencia, los resultados son mejores con una correcta planificación donde se incluyan tutorías que permitan el feedback alumno/profesor.

\section{Referencias}

BADILLO J. (2007) "La tutoría como estrategia viable de mejoramiento de la calidad de la educación superior. Reflexiones en torno al curso" en CUP-e Revista de Investigación Educativa 5.

BISQUERRA, R. (2002). La práctica de la orientación y la tutoría. Barcelona: Praxis

BISQUERRA, R. (2012). Orientación, tutoría y educación emocional. Madrid: Síntesis.

GELLALTY, A (1997) La inteligencia hábil. El desarrollo de las capacidades cognitivas. Buenos Aires: Ainque.

LILLO S., PÉREZ M., RODRIGO A., FERRER J.A. (2013) "La evaluación entre iguales en el marco de la tutoría grupal, estrategia docente para la mejora del proceso de enseñanza-aprendizaje en alumnos de primeros curso s. Dibujo Arquitectónico I. Grado de Arquitectura Técnica",en Actas XXI Congreso Universitario de Innovación Educativa en las Enseñanzas Técnicas (2013 Valencia), 1069 .

MONTES N., MACHADO E.F. (2011). "Estrategias docentes y métodos de enseñanza-aprendizaje en la Educación Superior" en Humanidades Mededicas vol.11 no.3. 